(c) American Dairy Science Association, 2004.

\title{
Measurement of Gas Holes and Mechanical Openness in Cheese by Image Analysis*
}

\author{
M. Caccamo, ${ }^{1}$ C. Melilli, ${ }^{1}$ D. M. Barbano, ${ }^{2}$ G. Portelli, ${ }^{1}$ \\ G. Marino, ${ }^{1}$ and G. Licitra ${ }^{1,3}$ \\ ${ }^{1}$ CoRFiLaC, Regione Siciliana, \\ 97100 Ragusa, Italy \\ ${ }^{2}$ Northeast Dairy Food Research Center, \\ Department of Food Science, \\ Cornell University, Ithaca, NY 14853 \\ ${ }^{3}$ Dipartimento di Scienze Agronomiche, \\ Agrochimiche e delle Produzioni Animali, \\ Catania University, Via Valdisavoia 5, \\ 95100 Catania, Italy
}

\begin{abstract}
A method to measure the amount of the surface area of cheese slices occupied by gas holes was developed to reflect the relative gas production among different cheeses. A digital camera mounted on a copy stand with lighting was used to make digital images of each slice of cheese. A commercial digital image analysis software program was used and an algorithm was written to measure the area of the image of the cheese slice occupied by holes. The image was cropped and scanned to determine which color channel produced the best image contrast. The MATLAB ${ }^{\circledR}$ program allowed the user to eliminate mechanical openness or false holes and then to scan the image to produce a percent distribution of pixels in the image as a function of pixel intensity. The user then determined a threshold value to differentiate pixels that were in holes from those representing areas with no holes. The percentage of the total surface area occupied by holes was calculated. The coefficient of variation of the method ranged from $2.43 \%$ with gas holes of about $1 \%$ of the surface of the cheese slice to a coefficient of variation of $0.92 \%$ with gas holes of about $6.8 \%$ of the surface area of the cheese slice. Examples of applications of this method are given for Emmental, Ragusano, and Cheddar cheeses. The method can be used as a tool in research studies to correlate the amount of gas production with manufacturing condi-

Received July 14, 2003

Accepted October 6, 2003.

Corresponding author: D. M. Barbano; e-mail:dmb37@cornell.edu.

*Use of names, names of ingredients, and identification of specific models of equipment is for scientific clarity and does not constitute any endorsement of product by authors, Cornell University, the Northeast Dairy Foods Research Center, CoRFiLaC, or Dipartimento di Scienze Agronomiche, Agrochimiche e delle Produzioni Animali, Catania University.
\end{abstract}

tions or as a quality control tool in cheese manufacturing.

(Key words: image analysis, gas production, mechanical openness)

\section{INTRODUCTION}

Gas production in some cheese varieties (e.g., Swiss style cheeses) is part of the typical characteristic of the cheese (Rheinbold, 1972), while in other cheese varieties (e.g., Cheddar) gas production is considered to be a defect (Fox et al., 2000). Generally, gas production produces holes or cracks in the structure of cheese that can vary in number, distribution, size, and shape. White et al. (2003) indicated that one of the least controlled defects in round eye cheeses is development of splits that appear during refrigerated storage after cheese is removed from the warm room. In other cheese varieties (e.g., Gouda and Ragusano), a small amount of gas production is typical. Generally, abnormal gas production in cheese has been divided into 2 types: early gas and late gas production (Kosikowski and Mistry, 1997). Early gas is typically produced by coliforms, while late gas production in cheese is typically caused by Clostridium tyrobutyricum (Kosikowski and Mistry, 1997). In Cheddar cheese, citrate-fermenting lactobacilli are thought to be the cause of gas production that leads to slit formation (Fox et al., 2000).

Digital imaging technology has been applied to the determination of the distribution, size, and color of blisters on pizza (Yun et al., 1994) and to determine length of shreds of Mozzarella cheese (Apostopoulos and Marshall, 1994; Ni and Gunasekaran, 1998). Modern digital imaging technology has been developing rapidly and offers an opportunity to rapidly quantify and characterize gas production in cheese by taking photographs with a digital camera and using commercial image analysis software. Recently, image analysis has been applied 
for analysis of SEM images of cheese microstructure (Pastorino et al., 2003). The objective of the present study was to develop an image analysis method to measure the area of the surface of cheese slices occupied by gas holes (or other types of mechanical openness) for use in measurement of gas production in research studies and for quality control in cheese manufacturing.

\section{MATERIALS AND METHODS}

\section{Photography and Image Analysis}

An overview of the image analysis method is summarized in the following steps: 1) cut slices of cheese of uniform thickness (from slice to slice and within slice) to be photographed; 2) set the conditions (lamp intensity and angle, background, camera distance from surface of cheese, etc.) and photograph the slice of cheese with a black background; 3) make digital photo and import it into the computer; 4) manually crop the image; 5) use image analysis software to select the RGB (red, green, blue) channel that provides the best contrast between areas of the image with and without holes; 6) if measurement of holes due only to gas production is desired, then the user identifies and manually eliminates holes that are due to mechanical openness and not due to gas by selecting these locations on the image, and the program converts all the pixels in that location to an intensity of $255 ; 7)$ scan the image and display a graph of percentage of total pixels as function of pixel intensity (from 0 to 255); 8) identify the threshold pixel intensity to separate pixels (lower than the threshold) representing holes from those representing areas with no holes; 9) stretch all pixels less than or equal to the threshold to 0 and all those greater than the threshold to 255 to produce a black and white image of the cheese slice; 10) calculate the black pixels as a percentage of the total of black plus white pixels; and 11) compare to position of holes on the black and white image with the original image to visually verify that the threshold is correctly identifying the holes. This general approach is similar to that applied by Pastorino et al. (2003) to analysis of SEM images of the microstructure of cheese.

Cheese slices were placed, one by one, on the copy stand table with a black background and a photograph of each slice was taken using a digital camera (Nikon Coolpix E 990, model no. 4112962, 3.34 Megapixel, Nikon Corporation, Tokyo, Japan). The thickness of the slice of cheese needs to be optimized for the particular type of cheese and the average size of gas holes in that type of cheese. Examples of this are given later in this paper. The camera was set at macro, quality basic VGA, and the flash was off. The camera was mounted on a copy stand at $23 \mathrm{~cm}$ from the surface of the table. The slice of cheese was illuminated by a lamp (Rotomatic, model 1000 Zoom, lamp OSRAM Supershot V230W1000, Germany) positioned to avoid shadows on the surface of the cheese slice. Each photograph had a resolution of $640 \times 480$ pixels and was saved as a jpg file. This resolution was selected to reduce the time of image analysis (approximately 1 to 2 min per image), to have a lower level of grey, and to increase contrast.

The digital image file was analyzed using MATLAB (Version 6.0.0.88, 2000, The Mathworks, Inc., Asheboro, NC). An algorithm was written and implemented in MATLAB. The image file was opened in the MATLAB program and the user manually cropped the image to eliminate everything not to be scanned (i.e., labels, background, etc. as in Figure 5a, 5b, and 5c). The image was processed as a three-dimensional matrix of values to determine the intensity of red, green, and blue (RGB) channel of each pixel. The program let the user choose the channel (i.e., red, green, or blue) that produced the best contrast between holes and areas of the cheese with no holes. The best channel may differ from one type of cheese to another, or it may differ from one set of photographic conditions to another. However, in one experiment for one cheese type analyzed under the same photographic conditions, the best channel should be selected and used consistently for all images. For the cheeses analyzed in the present study, the blue channel was best for the Ragusano and Emmental cheeses, while the red channel was best for the Cheddar cheese that contained added annatto coloring. The analysis of the image to measure the percentage of the total area occupied by gas holes started from this point. First, if only a measurement of gas holes is desired, then the holes that were due to mechanical openness and not gas were identified and manually eliminated by selecting these locations on the image and having the program convert all the pixels in that location to an intensity of 255. Next, the image was scanned, and a graph of percentage of total pixels as function of pixel intensity (from 0 to 255) was displayed. An example of a pixel intensity plot is given in Figure 1a. The portion of this graph at the onset of the peak representing pixels where there were no holes present was expanded to determine the pixel intensity threshold (Figure 1b). The threshold was defined as the point in the baseline on the lower pixel intensity side of the pixel intensity peak representing the point where there was a significant and consistent increase in the slope of the baseline that reflected the onset of the peak (Figure 1b). The threshold value was used to divide the pixels into those representing holes (i.e., less than or equal to the threshold) vs. pixels not representing holes. Next, the program stretched the pixel intensity for all pixels less than or equal to the threshold to an intensity of 0 and all those greater than the threshold to an intensity of 255 to 

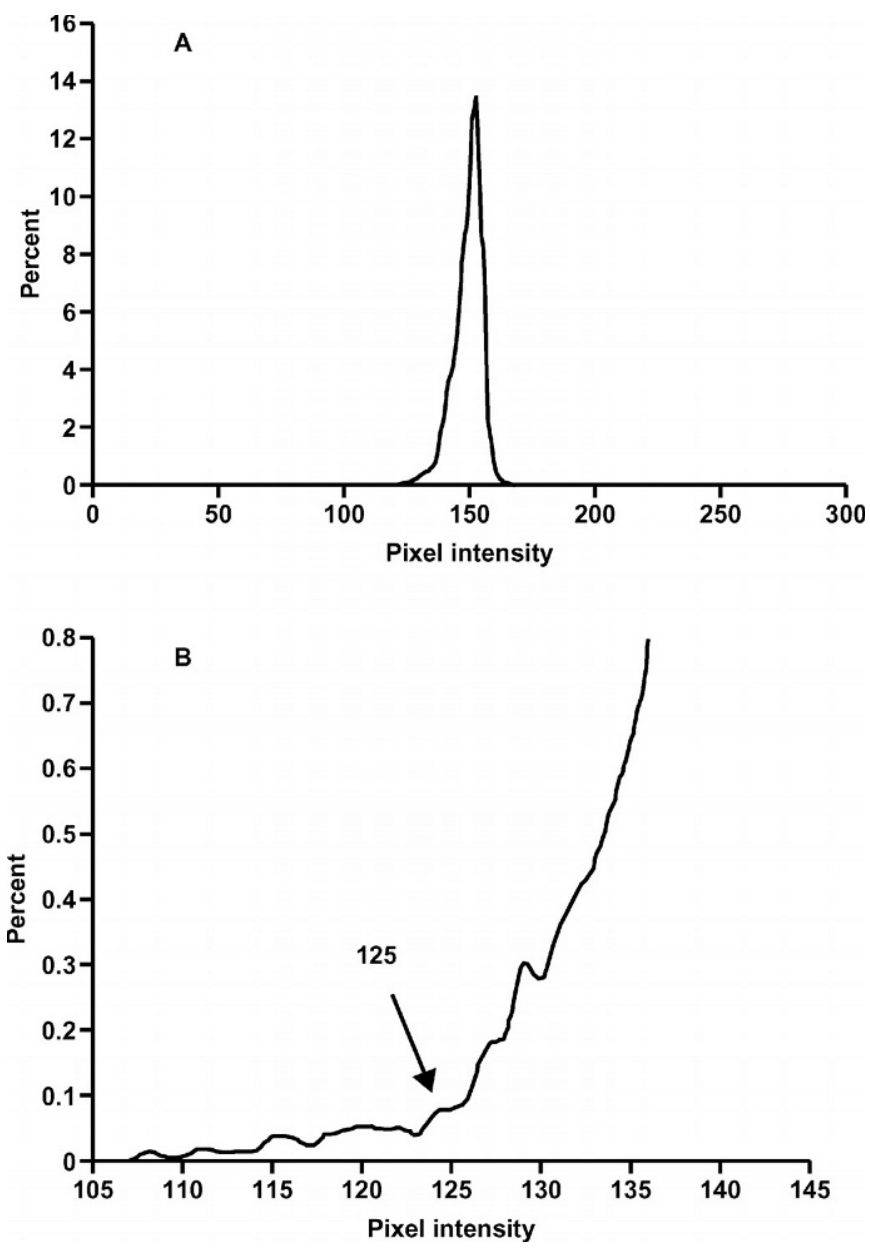

Figure 1. Example of a pixel intensity plot (a) and an expanded view to identify pixel intensity threshold (b).

produce a black and white image of the cheese slice (Figure 2a), which was compared with the original photo of the cheese slice (Figure $2 b$ ) to ensure that the program identified holes correctly. Comparison of the large gas hole at the left edge of Figures $2 a$ and $2 b$ demonstrates the issue of illumination, shadows, and size of hole. In Figure 2b the hole can be seen clearly, but the shadowing within the hole is not uniform. More light is being reflected at the lower half of the hole than the top half. Thus, only about $80 \%$ of the original hole was detected (Figure 2a). The black pixels as a percentage of the total of black plus white pixels was calculated and expressed as the percentage of holes in the cheese slice.

The method of selection of the threshold pixel intensity value to separate pixels that represent holes from those not representing holes is a critical step. We tried several methods of automating the selection of the threshold value in the program. However, variation in characteristics of the plot of the relative percentage of pixel intensity from cheese slice to cheese slice, particularly when there was a wide range of gas production and hole sizes, made the automation of threshold determination difficult. The variations observed were: multiple peaks (some representing areas of cheese surface with no holes but slightly different in color or shadowing due to variation in lighting or thickness of the slice and some representing holes), skew of the pixel intensity peaks, and high background level of pixel at lower intensities due to a large number of holes of varying depth and shadowing.

The situation is very simple for a slice of cheese that has a low percentage of holes or no holes (Figure 1a). There is one major peak in the percent distribution in the pixel intensity plot and the threshold needs to be at the onset of that peak. A high and variable level of pixel intensities lower than the mean (i.e., 10 to 100) of the main peak in the pixel intensity distribution caused by a large number of small holes of varying size and depth, makes an automated determination of the threshold more difficult. A different situation occurs when there is a small number of large holes that allow the black background to completely or partially show through the hole (Figure 3a). This is often the case for an Emmental cheese. In this case there can be 2 (Figure $3 a$ ) or more peaks (Figure 3b) in the plot of the distribution of pixel intensities. When this happens it is necessary for the user to determine which peaks in the pixel intensity graph represent holes and which peaks represent cheese without holes. This is done by making multiple scans of the original image (Figure 3c), selecting a different threshold value in successive scans, and comparing the black and white image of areas showing what the program defined as holes, vs. no holes, with the original photograph. In Figure 3c, the original photo of the slice of cheese can be compared with the black and white image to determine if the areas the program defined as holes and cheese using a threshold value of 100 (Figure 3d) and 85 (Figure 3e) are correctly representing the holes. In the case of the threshold value at 100 (Figure 3d), the program has incorrectly identified all of the bottom left corner of the slice as a hole, and that is not correct based on viewing the original photo (Figure 3c). This is probably due to the slice being slightly thinner in that area. With a threshold value of 85 (Figure 3e), the program has correctly identified the small hole in the lower left corner of the image and the two large holes. This is an example of how the operator can verify the correctness of the value selected for the threshold.

\section{Evaluation and Application of the Method}

The image analysis method was evaluated for accuracy and ability to detect differences among images 

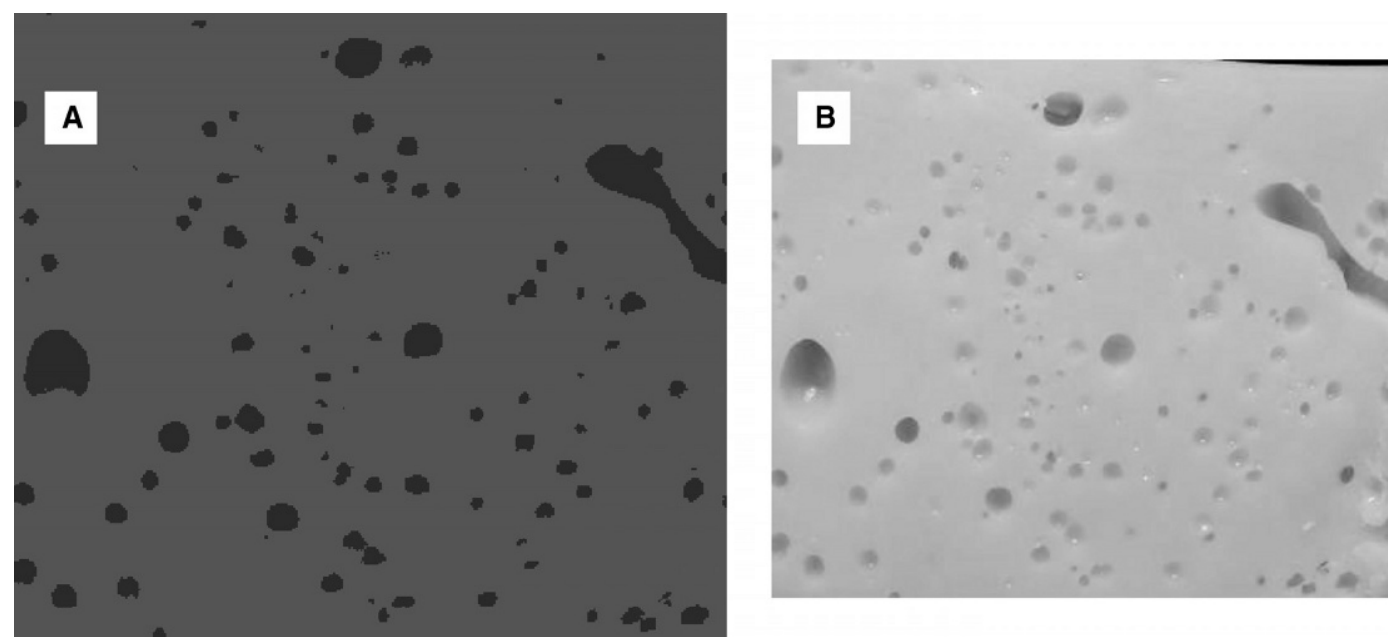

Figure 2. Gray scale image of a cheese slice (a) compared with the original photo (b).

with a range of hole areas that represents a normal level of gas that would be considered a mild to strong defect in Ragusano cheese. Microsoft Paint was used to make a series of 6 images with a light yellow background with black spots (to simulate holes) at 6 different area levels that could be encountered in scanning Ragusano cheese slices that had gas defects. The $6 \mathrm{im}-$ ages were saved as separate files in bitmap format and printed on a color printer. Each image was photographed under the same conditions used to photograph slices of cheese. The size of the printed images was about $8.73 \times 8.73 \mathrm{~cm}$, and black circles on the yellow background were uniform in size (approximately 0.44 $\mathrm{cm}$ in diameter). All black circles were completely within the yellow background and none overlapped the edge. The diameter of each black spot was measured using a caliper that had a graduation every $0.05 \mathrm{~mm}$, and the percentage of the total area in each of the 6 slices that was represented by black spots was calculated. This was replicated 3 times for each of the 6 images. The value obtained by measurement with a caliper and calculation was compared with the values determined by scanning the photographic images with the MATLAB program. Each of the six images was cropped and analyzed 3 times with the MATLAB program. The data were analyzed using the GLM procedure of SAS using an ANOVA model with terms for method $(n=2)$, image $(n=6)$, and replicate $(n=3)$ and interaction terms. The mean results of the 2 methods and between images were compared using a $t$-test $(P$ $\leq 0.05)$.

The repeatability of the image analysis method using MATLAB was determined by selecting 3 cheese images for 1 cheese type, with different degrees of gas production, cropping and scanning each image 6 times and calculating the mean, standard deviation, and coefficient of variation. The ability of the method to determine significant differences in amount of gas production among these 3 Ragusano cheeses with 3 different levels of gas production was determined by cropping and scanning each of the 3 images 6 times. The data were analyzed with the GLM procedure of SAS using an ANOVA model with terms for image $(\mathrm{n}=3)$ and scan $(\mathrm{n}=6)$. The difference in the amount of gas holes among images was considered to be different if $P \leq 0.05$ for both the model and image.

Three different varieties of cheese (Ragusano, Emmental, and Cheddar) were selected to demonstrate the application of the method to different characteristics of abnormal and normal gas production in cheese. These cheeses were selected because the method works best with cheeses that can be sliced to a uniform thickness, that form slices with a smooth surface, and have sufficient slice flexibility so they do not break during cutting and handling. For Ragusano and Cheddar, a slice thickness of $1 \mathrm{~cm}$ was used. However, for Emmental cheese with larger gas holes a slice thickness of $1 \mathrm{~mm}$ produced better definition of gas holes. The Ragusano and Emmental cheese slices were $10 \times 10 \mathrm{~cm}$, whereas the Cheddar slices were $10 \times 20 \mathrm{~cm}$.

\section{RESULTS AND DISCUSSION}

\section{Scanning Accuracy}

The accuracy of the scanning process was checked by comparison of the area of black circles on a yellow background as a percentage of total image area measured by caliper with the area of black as a percentage of total image area calculated by MATLAB (Table 1). 

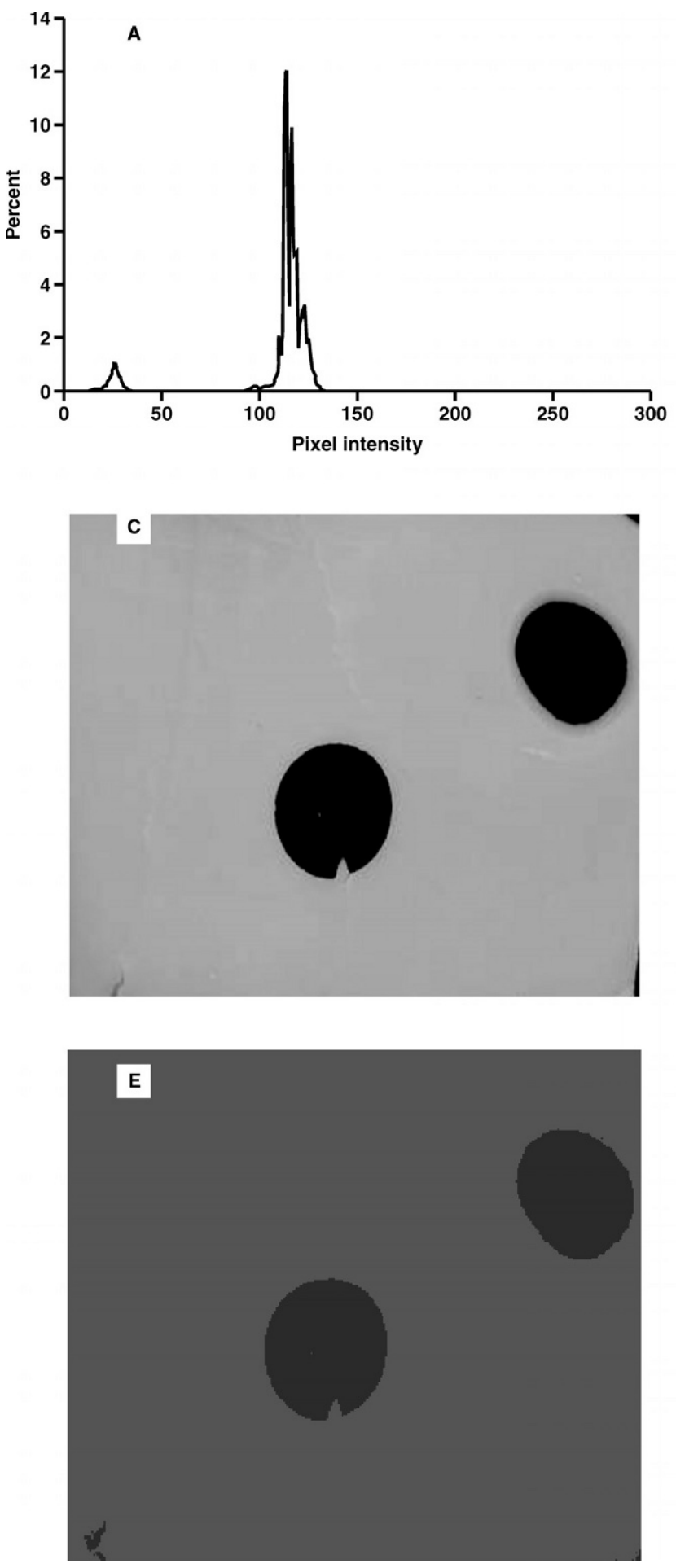

Figure 3. (a) Pixel intensity plot of Emmental cheese with the peak at 30 representing the black background showing clearly through a large hole, and the peak at 120 representing an area of cheese without holes, (b) peaks at 70 and 90 represent holes with different shading and the peak at 110 represents the areas of the cheese without holes, (c) an original image of a slice of Emmental cheese with 2 holes, (d) a grayscale image of the holes detected by MATLAB with a threshold value of 100 , and (e) a grayscale image of the holes detected by MATLAB with a threshold value of 85 . 
Table 1. Accuracy of measurement of the area of dark circles as a percentage of total area with a yellow background.

\begin{tabular}{lccl}
\hline Image & $\begin{array}{c}\text { Measured } \\
\text { by caliper }\end{array}$ & $\begin{array}{l}\text { Measured } \\
\text { by MATLAB }\end{array}$ & $\begin{array}{l}\text { Difference } \\
\text { MATLAB-caliper }\end{array}$ \\
\cline { 2 - 3 } 1 & & & \\
\cline { 2 - 3 } & 2.12 & 2.03 & -0.09 \\
2 & 4.31 & 3.88 & -0.43 \\
3 & 6.54 & 5.95 & -0.59 \\
4 & 8.20 & 7.73 & -0.47 \\
5 & 10.20 & 9.65 & -0.55 \\
6 & 12.15 & 12.06 & -0.09 \\
Average & $7.25^{\mathrm{a}}$ & $6.88^{\mathrm{b}}$ & -0.37 \\
\hline \multicolumn{2}{c}{${ }^{\mathrm{a}, \mathrm{b}}$ Means not sharing a common superscript differ $(P<0.05) . \mathrm{LSD}=$}
\end{tabular}
$0.07 \%$.

There was a difference $(P<0.05)$ in mean value for the caliper measurement vs. the MATLAB with the MATLAB giving slightly lower results. The exact reason for this difference was not determined, but the authors hypothesize that at the interface between the yellow and black of each black circle there is a transition zone of pixels intensities. When using a caliper and viewing the boundary between yellow and black by eye, a person will place the caliper at the outer edge of the zone of transition from black to yellow and thus may over estimate the diameter of the circular spot because a person uses the exterior-most dark portion of the image. The MATLAB image scanning counts individual pixels and their intensity independent of their position inside, at the interface, or outside of a black circle. In this case, if there was a gradient of black and yellow pixels at the edge of black circle, then it would be expected that using a caliper would overestimate the area
Table 2. Repeatability (mean, standard deviation, coefficient of variation) of MATLAB estimates of the percentage of gas holes for 3 different slices of Ragusano cheese with three different levels of gas production: (A) about $0.9 \%$, (B) about $2.6 \%$, and (C) about $6.8 \%$ gas holes.

\begin{tabular}{llll}
\hline Scan & $\begin{array}{l}\text { Image A } \\
\# 1547\end{array}$ & $\begin{array}{l}\text { Image B } \\
\# 1543\end{array}$ & $\begin{array}{l}\text { Image C } \\
\# 1549\end{array}$ \\
\hline & & $\%$ & \\
\cline { 2 - 4 } 1 & 0.953 & 2.555 & 6.898 \\
2 & 0.915 & 2.607 & 6.823 \\
3 & 0.964 & 2.718 & 6.898 \\
4 & 0.909 & 2.575 & 6.729 \\
5 & 0.935 & 2.689 & 6.824 \\
6 & 0.930 & 2.639 & 6.853 \\
Mean & $0.934^{\mathrm{c}}$ & $2.631^{\mathrm{b}}$ & $6.838^{\mathrm{a}}$ \\
SD & 0.021 & 0.064 & 0.063 \\
CV & 2.433 & 2.277 & 0.919 \\
\hline
\end{tabular}

${ }^{\text {a,b,c }}$ Means not sharing a common superscript differ $(P<0.05)$.

relative to MATLAB. Both methods (when the ANOVA was run on the data for each method independently) were able to distinguish a significant difference $(P<$ $0.01)$ among all 6 images. The LSD $(P<0.05)$ for the caliper method was $0.16 \%$ and for the MATLAB method was $0.20 \%$. Thus, both methods were very good in detecting differences that would be of practical importance in cheese making. The slightly higher variation within the MATLAB method than within the caliper method may be due to variation from replicate to replicate in the cropping of an image in the MATLAB method.

\section{Repeatability}

Images of 3 different slices of Ragusano cheese that demonstrated different amounts of gas development are
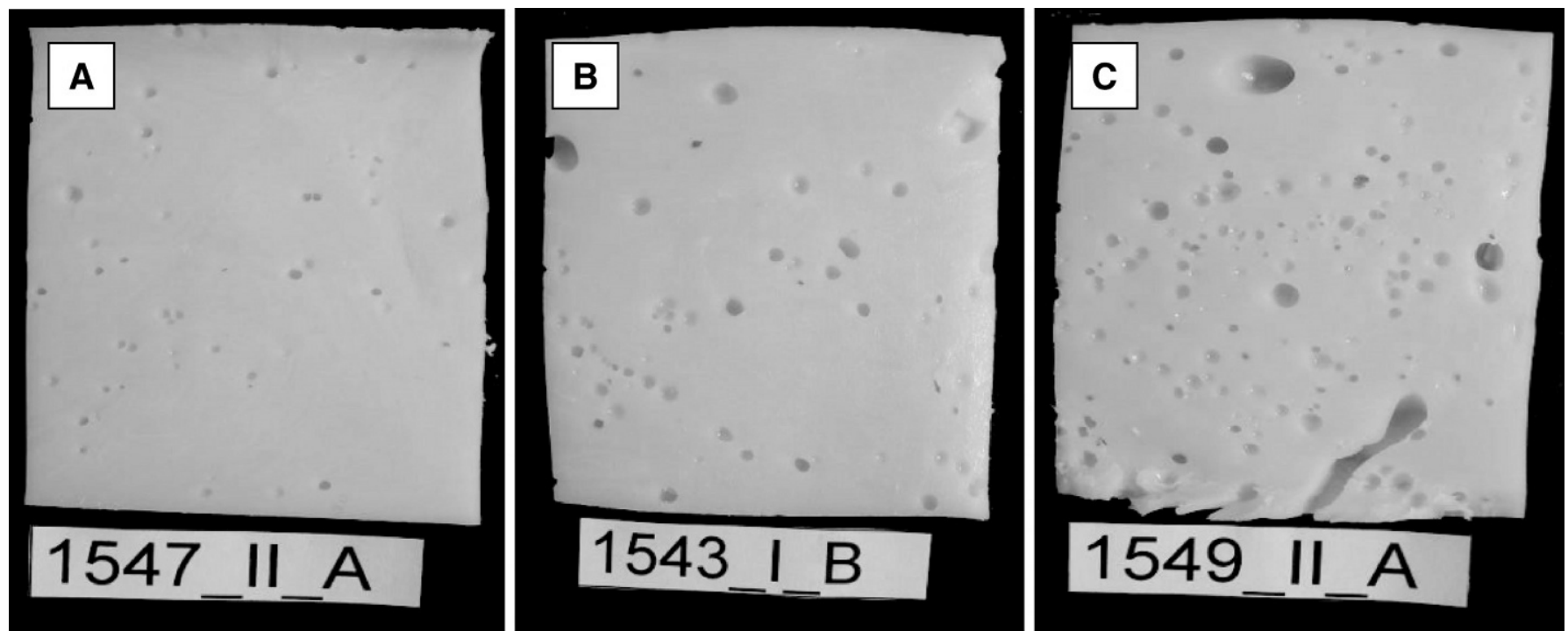

Figure 4. Three slices of Ragusano cheese from different blocks with different levels of gas production: (a) $0.9 \%$, (b) $2.6 \%$, and (c) $6.8 \%$. 

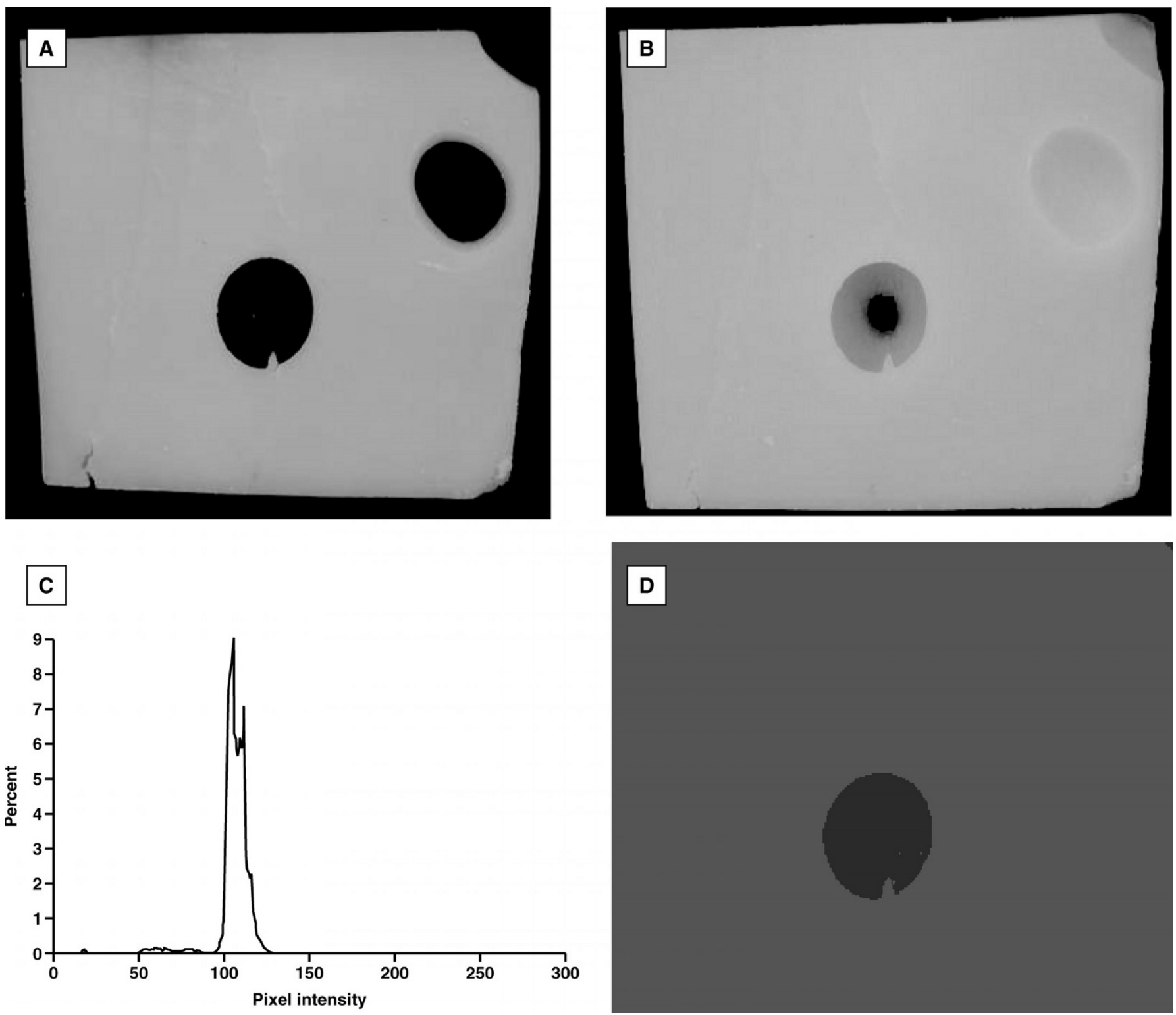

Figure 5. Photograph of 2 slices of Emmental cheese of different thickness: (a) $1 \mathrm{~mm}$ thick and (b) 10 mm thick, (c) pixel intensity plot of the $1 \mathrm{~mm}$ thick slice, and (d) grayscale image indicating the detection of holes in the cheese pictured as Figure 5b using a threshold value of 90 .

shown in Figure 4. In general, excessive early gas development in Ragusano cheese is undesirable. A few small gas holes are normal, as in Figure 4a, but the level of gas production seen in Figure $4 \mathrm{c}$ is unacceptable. The data for each of 6 scans of the 3 images in Figure 4 are shown in Table 2. The average percentage of holes for the 3 slices of cheese was about $0.9,2.6$, and $6.8 \%$ of the total area. Differences in the level of gas production are easily recognized by looking at the pictures, however the exact level of gas production and the amount of difference between different batches of cheese is not as easy to estimate by looking at the images. The image analysis system can provide a rapid quantitative measure of both level and differences among different pieces of cheese of the same type. As the total percentage of holes increased, the coefficient of variation decreased to approximately $0.9 \%$ when the proportion of the total area of the cheese had a level of gas production that represented $6.8 \%$ of the area of the cheese surface. The statistical analysis found a significant difference $(P<$ 0.05 ) among the 3 images (Table 2) and no significant differences among scans of the image. Thus, the method 
has sensitivity to measure differences in gas production and this technique could be useful as a simple quantitative method to study factors that influence the amount of gas production in cheese.

\section{Application to Different Cheese Varieties}

In Emmental cheese, gas production is a normal and desirable characteristic of the cheese when the size, number, distribution, and shape of the gas holes is correct (Reinbold, 1972). In Figures 5a and 5b, 2 images of Emmental cheese slices of different thickness are shown. In general, cheeses with larger diameter gas holes (in this case about $20 \mathrm{~mm}$ ) need to be prepared as thinner slices to achieve a better contrast between holes and background. In Figure 5a the slice was approximately $1 \mathrm{~mm}$ thick, while in Figure 5b the slice was about $10 \mathrm{~mm}$ thick. The photo in Figure 5b does not scan well because the gas hole in the upper right portion of the image is not cut in a way that the black background shows through the hole. The percent distribution of pixel intensity for the image in Figure 5b is shown as Figure 5c. When the threshold pixel intensity was set at 90 , the hole in the upper right portion of the image was not detected (Figure 5d). Adjusting the threshold to lower values did not allow the program to detect the hole that did not go completely through the slice. The pixels inside this hole were of an intensity that was within the range of pixel intensity from 100 to 130 , so they could not be separated from the areas with no holes by changing the threshold. A large-diameter hole that is not very deep will not have a shadow effect from the lighting that allows the program to differentiate that area of the cheese slice as a gas hole. It would be possible for the user to manually "paint" the hole in another program, but this would be very time consuming. When painting the hole was done, it produced the percent distribution shown in Figure $3 \mathrm{~b}$ with 3 intensity peaks. The first peak represents the hole in the center of the slice at an intensity value of 60 , the second peak at an intensity value of 90 represents the hole that was painted to allow it to be detected, and the third peak at 120 represents the surface area of the cheese without holes. In contrast to large shallow holes, the holes of smaller diameter have more depth and less width (as in Figure 4), and shadows are produced from the angle of incident lighting that allows the program to correctly identify and measure the area of the holes. Image analysis could be a useful tool for a quality assurance program to provide a quantitative measure of the normal amount of gas production, variation in the amount of gas production across time, and abnormal shape or distribution of eyes throughout the structure of

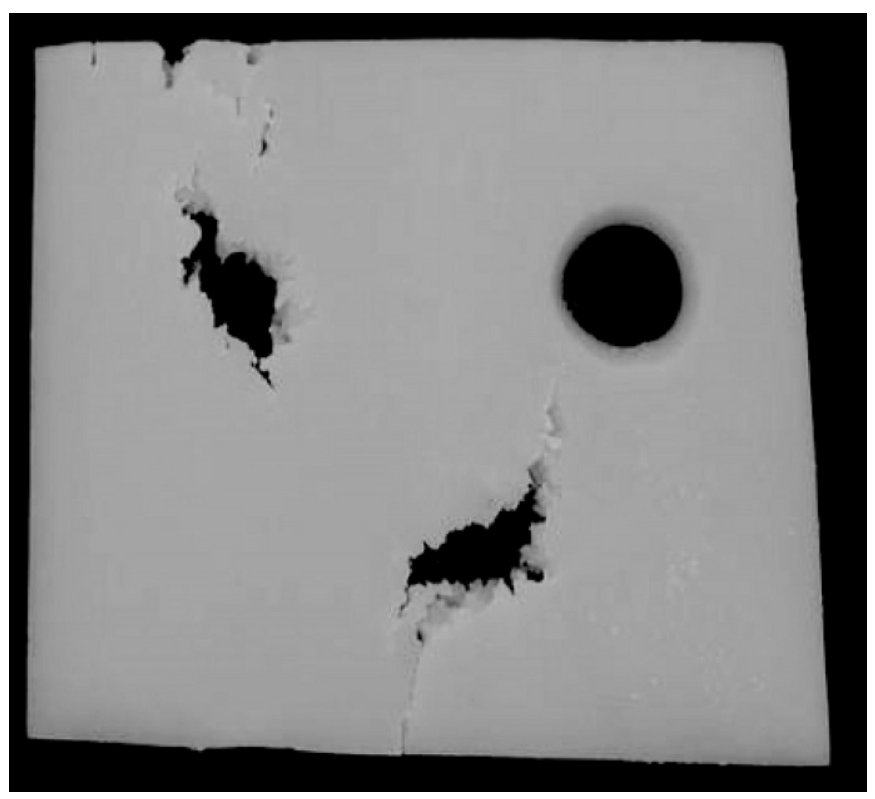

Figure 6. Example of a normal gas hole and abnormal gas hole, or mechanical openness, in Emmental cheese.

a cheese such as Emmental. In Figure 6, a photographic image of a slice of Emmental demonstrates the presence of both a normal and abnormal gas formation. The normal gas formation represents $3.51 \%$ of the area and the abnormal gas hole formation represents $3.71 \%$ of the area. A photographic image could be captured at an image capture station located at a cheese cutting line, transmitted to a computer, and scanned. The evaluation of the characteristics of gas production could be recorded and correlated to conditions in the cheese manufacturing process.

Cheddar cheese should not have gas defects. However, a gas defect that commonly develops in aged Cheddar cheese is slits (Figure 7). This defect manifests itself at about 90 to $120 \mathrm{~d}$ of aging. The image analysis approach was capable of distinguishing slits from areas with no slits and could provide a quantitative estimate of the percentage of area represented by slits. While the percentage of the total area of the cheese that is represented by slits is a relatively small (e.g., in Figure $7 \mathrm{a}$ equals $0.85 \%$ of the area as slits and Figure $7 \mathrm{~b}$ equals $0.65 \%$ of the area as slits), the structure of the cheese is weakened and when the blocks of cheese are cut into chunks of 0.25 to $1 \mathrm{~kg}$ on automated cutting systems, the chunks break and cheese is lost to trim, which has a lower value. A systematic scanning of images of Cheddar cheese structure and evaluation of the frequency of occurrence of slits could be a useful tool to detect small changes in frequency of development of this defect in routine production, and small but important changes 

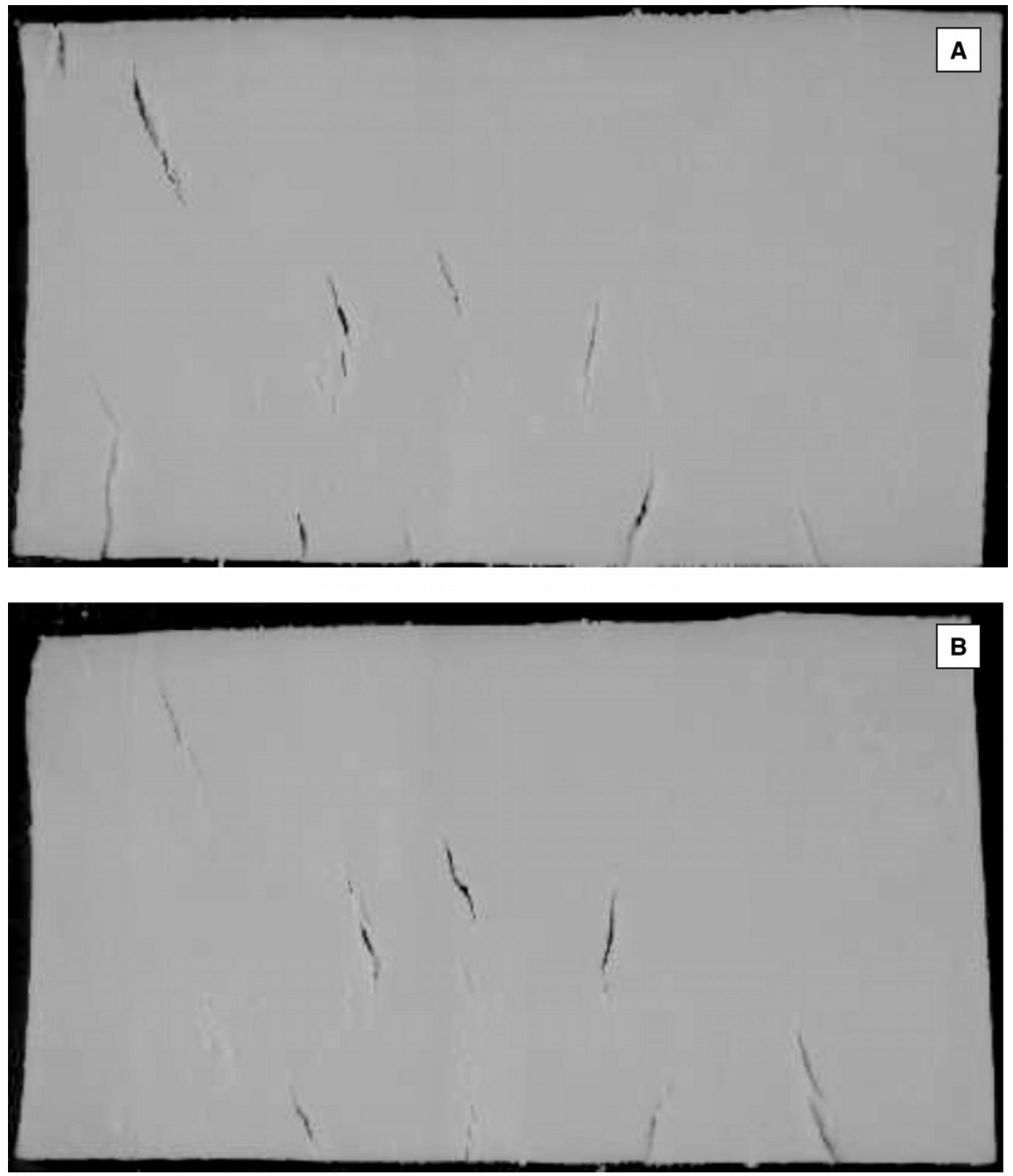

Figure 7. Cheddar cheese with slit defect: (a) $0.85 \%$ of the area as slits and (b) $0.65 \%$ of the area as slits.

in the percentage of slits could provide a signal for corrective actions to be taken at the manufacturing level before the severity of the defect causes large economic losses.

\section{CONCLUSIONS}

A digital photographic and software image analysis approach was developed to provide repeatable measurement of the area of the cheese structure represented by gas holes and/or mechanical openness and to be able to detect relative differences among cheeses analyzed under the same conditions. The commercial availability of digital photographic equipment and image analysis software packages makes it possible for image analysis to be used in both research and in industrial quality control in cheese manufacture. In research, factors influencing the level and characteristics of gas production, as it relates to the appearance of the cheese can be rapidly measured. For quality assurance in cheese manufacturing, routine data can be collected to describe quantitatively the frequency and characteristics of both 
normal and abnormal gas production or openness in cheese structure. These measurements could be used as a basis for management decisions to control and improve cheese quality.

\section{ACKNOWLEDGMENTS}

The authors thank Sebastiano Campo, Patrizia Campo, and Elena Calabrese for technical assistance. Financial support was provided by the Assessorato Agricoltura e Foreste della Regione Siciliana, Palermo, Italy.

\section{REFERENCES}

Apostopoulos, C., and R. J. Marshall. 1994. A quantitative method for determination of shred quality of cheese. J. Food Qual. $17: 115-128$
Fox, P. F., T. P. Guinee, T. M. Cogan, and P. L. H. McSweeney. 2000. Pages 232 to 235 in Fundamentals of Cheese Science. Aspen Publishers, Gaithersburg, MD.

Kosikowksi, F. V., and V. V. Mistry. 1997. Pages 252 to 265 in Cheese and Fermented Milk Foods, Volume 1. F. V. Kosikowski, ed. L.L.C., Westport, CT.

Ni, H., and S. Gunasekaran. 1998. A computer vision method for determining the length of cheese. Artificial Intelligence Rev. 12:27-37.

Pastorino, A. J., C. L. Hansen, and D. J. McMahon. 2003. Effect of salt on the structure-functional relationships of cheese. J. Dairy Sci. 86:60-69.

Reinbold, G. W. 1972. Swiss Cheese Varieties. Pfizer Cheese Monographs. Volume 5. Pfizer, Inc., New York, NY.

White, S. R., J. R. Broadbent, C. J. Oberg, and D. J. McMahon. 2003. Effect of Lactobacillus helveticus and Proprionibacterium freudenrichii ssp. shermanii combinations on propensity for split defect in Swiss cheese. J. Dairy Sci. 86:719-727.

Yun, J. J., D. M. Barbano, E. F. Bond, and M. Kalab. 1994. Image analysis method to measure blister size and distribution on pizza. Pages 33 to 38 in Proc. 31st Annu. Marschall Italian Specialty Cheese Seminar. Madison, WI. 